BULLETIN OF THE

AMERICAN MATHEMATICAL SOCIETY

Volume 80, Number 1, January 1974

\title{
RATIONAL CRITICAL POINTS OF THE REDUCED NORM OF AN ALGEBRA
}

\author{
BY DON GOELMAN
}

Communicated by Joseph Rotman, July 13, 1973

1. Introduction. For a polynomial function $f: E \rightarrow k$, where $E$ is an $n$ dimensional vector space over the field $k$, the determination of $\left(C_{f}\right)_{k}$, the set of critical points of $f$ lying in $E$, has been of interest historically (see [5], and [7, Chapter 8]). More recently, its importance has been noted in the theory of Gauss transforms and zeta functions [6]. In particular, for the case $E=A$, a central simple algebra over an $A$-field $k$, the function of interest is the reduced norm $v: A \rightarrow k$; it is with respect to this polynomial that the more "classical" zeta function is defined (see [8, p. 203]).

The main result in this note is contained in the next section, where $\left(C_{v}\right)_{k}$ is determined for a (not necessarily central) simple algebra whose dimension over $k$ is not divisible by the characteristic of $k$. In the last section the critical set is determined for any associative algebra with unity, under certain separability conditions.

For the definition of the reduced representation and norm in the general (nonsimple) case, see [1]. For the classical structure theorems of algebras, see also [3] and [4]. The definition of critical sets is understood as in [6]. For a simple algebra $A$ over $k$, the the following notation has been adopted: $K$ is the center of $A$, so that $A=M_{m}(D)$, a full matrix algebra over the $K$-central division algebra $D$, the dimension of $D$ as a vector space over $K$ is $d^{2}$, and $[K: k]=t$; thus $d$ is the index of $A$ and $D$ over $K$; $r=m d$, the degree of $A$ over $K$; and $n=r^{2} t$, the (vector space) dimension of $A$ over $k$. We also have the reduced norms $v: A \rightarrow k, v^{\prime}: A \rightarrow K, v^{*}: D \rightarrow k$, and $\nu^{\prime \prime}: K \rightarrow k$ (which is, of course, the field norm here). For the general $k$-algebra $A$, we let $N$ denote its radical, and $A_{1}, \cdots, A_{s}$ the simple component summands of $A \mid N$, each with the appropriate invariants $K_{i}, D_{i}$, $m_{i}, d_{i}, r_{i}, t_{i}, n_{i}$, and reduced norms $\bar{\nu}: A \mid N \rightarrow k$ and $\nu_{i}: A_{i} \rightarrow k, i=1, \cdots, s$. Also $(d v)_{z}$ is the differential map of the norm at $z .(d v)_{z}(h)$ is the coefficient of $t$ in the polynomial $v(z+t h)-v(z)$, for $h \in A, t \in k ;(d v)_{z}$ is a $k$-linear functional.

AMS (MOS) subject classifications (1970). Primary 10M05, 12A80; Secondary 14G05.

Key words and phrases. Reduced norm, reduced representation, central simple algebra, critical set. 


\section{Simple algebras.}

THEOREM 1. Let $A$ be a simple algebra of dimension $n$ over a field $k$, where the characteristic of $k$ does not divide $n$. Then

$$
\left(C_{v}\right)_{k}=\{z \in A \mid \operatorname{rk}(z) \leqq \rho(A)\},
$$

where $\operatorname{rk}(z)$ is the left row-rank of $z$ as a matrix in $M_{m}(D)$, and $\rho(A)=$ $m-1$ or $m-2$, according as $d t=1$ or $d t>1$, respectively.

The proof is by escalation, starting with the most trivial cases.

Case I. Assume $m=t=1$. In other words, $A$ is a central division algebra. Then by the homogeneity of the norm and by Euler's formula, $\left(C_{v}\right)_{k} \subseteq\{z \in A \mid v(z)=0\}=\{0\}$. But 0 is a critical point in this case if and only if $d=1$.

Case II. Assume $t=1$. Then writing the elements of $A$ as $m \times m$ matrices over $D$, we can show, in the spirit of [2, p. 152], the following:

LEMMA. For all $z \in A$, with $\mathrm{rk}(z)=\rho \geqq 1$, there exist $b_{1}, b_{2} \in S L_{m}(D)$ such that $b_{1} z b_{2}$ is a diagonal matrix $\left[a_{i j}\right]$, with $a_{i j}=0$ for $i \neq j$ and for $i>\rho$, and $a_{11}, \cdots, a_{\rho \rho}$ nonzero.

Now the reduced norms $v: A \rightarrow k$ and $v^{*}: D \rightarrow k$ are related by this formula [8, p. 169]: If $z \in A$ is a triangular matrix with $z_{1}, \cdots, z_{m} \in D$ the diagonal entries, then $v(z)=v^{*}\left(z_{1}\right) \cdots v^{*}\left(z_{m}\right)$. In particular, by the lemma, $\operatorname{rk}(z) \leqq$ $m-1$ implies $v(z)=0$. Moreover, since $S L_{m}(D)$ is generated by the transvections, $v(z)=1$ for $z \in S L_{m}(D)$. Therefore, we can reduce this case to diagonal matrices, since $(d v)_{b_{1} z b_{2}}\left(b_{1} h b_{2}\right)=(d v)_{z}(h)$, for all $z, h \in A, b_{1}$, $b_{2} \in S L_{m}(D)$; i.e., $(d v)_{z}=0$ if and only if $(d v)_{b_{1} z b_{2}}=0$.

Since $m=1$ has been treated in Case I, we assume $m>1$, and let $z \in A ; 0<\operatorname{rk}(z)=\rho \leqq m$, with $z$ in the canonical form of the lemma, and with its diagonal entries $z_{1}, \cdots, z_{m}$. By the linearity of $(d v)_{z}, z \in(C v)_{k}$ if and only if $(d v)_{z}(h)=0$, for all $i, j$, for all $h$ in the $k$-space $W_{i j}=$ $\left\{\left(a_{r s}\right) \in A \mid a_{r s}=0,(r, s) \neq(i, j)\right\}$.

Now if $\rho=m, z$ is a unit and hence nonsingular. If $\rho \leqq m-2 ;$ then for all $i, j, h \in W_{i j}, \operatorname{rk}(z+t h) \leqq m-1$; so $v(z)=v(z+t h)=0$, and $(d v)_{z}(h)=0$.

Finally, if $\rho=m-1$ and $h \in W_{i j}$, with $(i, j) \neq(m, m)$, again $\operatorname{rk}(z+t h) \leqq$ $m-1$, so that $(d v)_{z}(h)=0$. However, if $h \in W_{m m}$,

$$
\begin{aligned}
v(z+t h)-v(z) & =v(z+t h)=v^{*}\left(z_{1}\right) \cdots v^{*}\left(z_{m-1}\right) v^{*}(t h) \\
& =t^{d} v^{*}\left(z_{1}\right) \cdots v^{*}\left(z_{m-1}\right) v^{*}(h) .
\end{aligned}
$$

Thus for $d>1,(d v)_{z}=0$, and if $d=1,0 \neq h \in W_{m m}$, then $(d v)_{z}(h) \neq 0$. This completes the treatment of Case II.

Case III. Assume only the hypothesis of the theorem. Then under the restriction on $n, K$ is a separable extension of $k$, and $v^{\prime \prime}(a)=\prod a^{\sigma}$, for 
$a \in K$, where $\sigma$ runs through the $k$-embeddings of $K$ in $\bar{k}$. We can then show that $v=v^{\prime \prime} \circ v^{\prime}$. Therefore $(d v)_{z}=d\left(v^{\prime \prime} \circ v^{\prime}\right)_{z}=\left(d v^{\prime \prime}\right)_{v^{\prime}(z)} \circ\left(d v^{\prime}\right)_{z}$. Hence $z \in\left(C_{v}\right)_{k}$ if and only if $\operatorname{im}\left(d v^{\prime}\right)_{z} \subseteq \operatorname{ker}\left(d v^{\prime \prime}\right)_{v^{\prime}(z)}$. Now $\operatorname{im}\left(d v^{\prime}\right)_{z}$ is a $K$-subspace of $K$; thus the last inclusion is equivalent to the following condition: Either $\operatorname{im}\left(d v^{\prime}\right)_{z}=\{0\}$, or $\operatorname{ker}\left(d v^{\prime \prime}\right)_{v^{\prime}(z)}=K$; in other words, $v^{\prime}(z) \in\left(C_{v^{\prime \prime}}\right)_{k}$ or $z \in\left(C_{v^{\prime}}\right)_{K}$. Since a modification of the argument from Case I yields the fact that $\left(C_{v^{\prime \prime}}\right)_{k}=\varnothing$ if $t=1$ and $\{0\}$ if $t>1$, we have, using this fact together with Case II, that $z \in\left(C_{v}\right)_{k}$ if and only if one of these conditions holds:

(i) $t>1$ and $\nu^{\prime}(z)=0$,

(ii) $d=1$ and $\operatorname{rk}(z) \leqq m-2$,

(iii) $d>1$ and $\operatorname{rk}(z) \leqq m-1$.

However, since $v^{\prime}(z)=0$ if and only if $\operatorname{rk}(z) \leqq m-1$, we can combine (i) and (iii) to prove the theorem.

\section{More general cases.}

THEOREM 2. Let $A$ be an associative algebra over $k$ with unity and let $N$ be its radical. Assume that the characteristic of $k$ does not divide any of the dimensions $n_{1}, \cdots, n_{s}$ of the simple components $A_{1}, \cdots, A_{s}$ of $A / N$. For $z \in A$, let $z+N=z_{1}+\cdots+z_{s}$, with $z_{i} \in A_{i}, i=1, \cdots$, s. Then $z \in\left(C_{v}\right)_{k}$ if and only if one of these conditions hold:

(I) There exists $i \neq j$ with $\mathrm{rk}\left(z_{i}\right) \leqq m_{i}-1$ and $\mathrm{rk}\left(z_{j}\right) \leqq m_{j}-1$.

(II) There exists $i$ with $\mathrm{rk}\left(z_{i}\right) \leqq \rho\left(A_{i}\right)$.

To prove this, we can first make the reduction to the semisimple case. We do this by noting that $z \in\left(C_{v}\right)_{k}$ if and only if $z+N \in\left(C_{\bar{v}}\right)_{k}$. For let $L$ be an algebraically closed field containing all the $K_{i}$. Then the absolute semisimplicity of $A \mid N$, following from our divisibility condition, implies in turn that $N \otimes L$ is the radical of $A \otimes L$, where all tensor products are over $k$. From here we can conclude that $\Lambda(z)=\Lambda^{\prime}(z+N)$, where $\Lambda$ and $\Lambda^{\prime}$ are the reduced representations of $A \otimes L$ and $(A \mid N) \otimes L$, respectively. The relation between $v$ and $\bar{v}$ follows from this.

We may therefore assume from now on that $A$ is already semisimple. Then we conclude again from the separability condition that $A \otimes L$ is semisimple. This implies [1, p. 121] that $\Lambda$ is an injection, and from here it follows that for all $z \in A$, with $z=z_{1}+\cdots+z_{s}, \Lambda(z)=\Lambda_{1}\left(z_{1}\right) \oplus \cdots \oplus \Lambda_{s}\left(z_{s}\right)$, where $\Lambda_{i}$ is the reduced representation of $A_{i}$. Hence $v(z)=v_{1}\left(z_{1}\right) \cdots v_{s}\left(z_{s}\right)$. Then if $h \in A$, with $h=h_{1}+\cdots+h_{s}, h_{i} \in A_{i}$, we have

$$
(d v)_{z}(h)=\sum_{i=1}^{s}\left[\left(d v_{i}\right)_{z_{i}}\left(h_{i}\right) \prod_{j \neq i} v_{j}\left(z_{j}\right)\right]
$$

for $z \in C_{v}$, then, since some $v_{i}\left(z_{i}\right)=0,(d v)_{z}(h)=\left(d v_{i}\right)_{z_{i}}\left(h_{i}\right) \prod_{j \neq i} v_{j}\left(z_{j}\right)$. Our result follows from here. 


\section{REFERENCES}

1. A. A. Albert, Structure of algebras, rev. ed., Amer. Math. Soc. Colloq Publ., vol. 24, Amer. Math. Soc., Providence, R.I., 1961. MR 23 \#A912.

2. Emil Artin, Geometric algebra, Interscience, New York, 1957. MR 18, 553.

3. E. Artin, C. J. Nesbitt and R. M. Thrall, Rings with minimum condition, University of Michigan Publ. Math., no. 1, Univ. of Michigan Press, Ann Arbor, Mich., 1944. MR 6, 33.

4. Max Deuring, Algebren, 2nd printing, Ergebnisse der Mathematik und ihrer Grenzgebiete, Band 41, Springer-Verlag, Berlin and New York, 1968. MR 37 \#4106.

5. P. W. Ketchum, Analytic functions of hypercomplex variables, Trans. Amer. Math. Soc. 30 (1928), 641-667.

6. Takashi Ono, Gauss transforms and zeta-functions, Ann. of Math. (2) 91 (1970), 332-361. MR 41 \#3436.

7. J. M. Wedderburn, Lectures on matrices, Amer. Math. Soc. Colloq. Publ., vol. 17, Amer. Math. Soc., Providence, R.I., 1934.

8. André Weil, Basic number theory, Die Grundlehren der math. Wissenschaften, Band 144, Springer-Verlag, New York, 1967. MR 38 \#3244.

Department of Mathematics, Villanova University, Villanova, Pennsylvania 19085 